\title{
Síndrome de Cotard asociado a Trastorno Depresivo Mayor con síntomas catatónicos. Informe de caso
}

\author{
Cotard Syndrome Associated to Major Depressive Disorder \\ with Catatonic Symptoms. Case report \\ Síndrome de Cotard associada ao transtorno depressivo \\ maior com sintomas catatônicos. Relato de caso
}

\author{
Daniel Mauricio Torrado-Arenas, MD * \\ Kevin Eduardo Santos-Gutiérrez, MD ** \\ Sandra Milena Ruiz-Higuera, MD *** \\ Laura Milena Zabala-Arias, $\mathrm{MD} * * * *$ \\ Jorge Andrés Niño-García, MD., Esp *****
}

\begin{abstract}
Resumen
Introducción: La catatonia es un síndrome neuropsiquiátrico con posturas anormales, mutismo y estupor. Tiene una prevalencia en Colombia del $11.4 \%$ de pacientes psiquiátricos. Objetivo: Discutir el curso clínico de una mujer de 34 años con antecedente de trastorno depresivo mayor que llega de urgencias con delirios nihilistas y síntomas catatónicos. Presentación del caso: Se reportó el caso de una adulta joven con trastorno depresivo mayor y síntomas psicóticos previamente hospitalizada hace nueve meses, egresó con tratamiento farmacológico que no recordaba. Llegó al servicio de urgencias del Hospital Universitario de Santander por tres días de comportamiento extraño, mutismo y negativismo. Paraclínicos y tomografía de cráneo normales. Se inició tratamiento con una benzodiacepina lográndose remisión de los síntomas catatónicos. Posteriormente refirió anhedonia, tristeza e ideas delirantes nihilistas; se consideró que correspondía a una recidiva del episodio depresivo que inició hace nueve meses ligado a síndrome de Cotard. Se adicionó sertralina con incremento
\end{abstract}

gradual hasta $150.0 \mathrm{mg}$ y olanzapina $5.0 \mathrm{mg}$ lográndose remisión de los síntomas afectivos y psicóticos. Discusión: El trastorno afectivo es la causa más frecuente de catatonia. Existen reportes similares, pero pocos donde coexistan las tres entidades; este es el primer caso reportado en el Hospital Universitario de Santander, con previo consentimiento informado. Conclusiones: Es inusual que un paciente deprimido presente delirios de negación y síntomas catatónicos simultáneamente, por lo cual este reporte es único y genera aporte a la literatura. Los síntomas catatónicos dificultan la exploración de otras esferas mentales y pueden ser secundarios a una condición médica, por lo cual es indispensable descartar organicidad, darles tratamiento oportunamente y así aclarar la psicopatología de base. [Torrado-Arenas DM, Santos-Gutiérrez KE, Ruiz-Higuera SM, Zabala-Arias LM, Niño-García JA. Síndrome de Cotard asociado a Trastorno Depresivo Mayor con síntomas catatónicos. Informe de caso. MedUNAB 2016;18(1): 76-80].

Palabras clave: Catatonia; Depresión; Negación (Psicología); Antipsicóticos; Trastornos psicóticos afectivos.

\footnotetext{
* Médico y cirujano, Universidad Industrial de Santander, Auxiliar docente de PAMRA-UIS, Miembro del grupo de investigaci\{on GINO-UIS.

** Médico y cirujano, Universidad Industrial de Santander

*** Médica Interna, Universidad Industrial de Santander

**** Médica y Cirujana, Universidad Industrial de Santander

***** Psiquiatra, Director del departamento de salud mental, Universidad Industrial de Santander, Bucaramanga. Santander
}

Correspondencia: Daniel Mauricio Torrado-Arenas. torradoarenasdanielmauricio@hotmail.com. Dirección: Carrera 29 \# 96 - 78 conjunto Miradores de San Lorenzo, Torre 16, Apartamento 302. Floridablanca-Santander 


\section{Abstract}

Introduction: Catatonia is a neuropsychiatric syndrome with abnormal postures, mutism and stupor. Colombia has a prevalence of $11.4 \%$ of psychiatric patients. Objective: To discuss the clinical curse of a 34-year-old woman with major depressive disorder that presents to emergency department with nihilistic delirium and catatonic symptoms. Case presentation: A young woman with history of unipolar major depression with psychotic features was hospitalized nine months ago. She was medicated with a nonspecific medical treatment. At the admission the patient had three days of bizarre behavior, mutism and negativism. Paraclinics and brain computer tomography didn't report any abnormality or changes. Treatment began with benzodiazepine, which achieved full remission of catatonic symptoms. After this, she developed anhedonia, sadness and nihilistic delusions and was considered as a relapse of a previous depressive episode from nine months ago, associated with Cotard's syndrome. Sertraline was added with gradual increase to $100 \mathrm{mg}$ and $5 \mathrm{mg}$ of olanzapine, getting a complete remission of psychotic and mood symptoms. Discussion: Affective disorders are most common cause of catatonia. There has already been a history of similar reports, but in few times this three entities were associated; this is the first case reported in Hospital Universitario de Santander, with informed consent. Conclusions: It is unusual for a depressed patient to present denial delusions and catatonic symptoms simultaneously; therefore this case is unusual and may contribute to literature. The catatonic symptoms make it difficult to explore other mental spheres, though they may be secondary to a medical condition, therefore, it is essential to dismiss organic pathologies and give initial treatment, so we can discover the underlying etiopsychopathology. [Torrado-Arenas DM, SantosGutiérrez KE, Ruiz-Higuera SM, Zabala-Arias LM, NiñoGarcía JA. Cotard's syndrome associated to major depressive disorder with catatonic symptoms. Case report. MedUNAB 2016;18(1): 76-80].

Keywords: Catatonia; Depression; Denial (Psychology); Antipsychotic Agents; Affective Disorders, Psychotic.

\section{Introducción}

El síndrome de Cotard y el síndrome catatónico no están incluidos como entidades nosológicas independientes en los sistemas de clasificación psiquiátrico, pero se pueden presentar en el contexto de enfermedades categorizadas como el trastorno depresivo $(1,2)$. La catatonia es un síndrome caracterizado por alteraciones motoras, en conciencia, afecto y pensamiento; cuyos tres signos cardinales son posturas anormales, mutismo y estupor $(3,4)$. En Colombia se reporta una prevalencia de catatonia de $11.4 \%$ en pacientes psiquiátricos hospitalizados, comparados con $10.0 \%$ a nivel mundial $(3,5)$. Otros estudios han reportado la presencia de sintomatología mixta (depresión y manía) precedido la catatonia hasta en el 50.0\% de los casos, así como comorbilidad con esquizofrenia hasta en un $15.0 \%(1,6)$. Las causas más importantes de catatonia

\section{Resumo}

Introdução: A catatonia é uma síndrome neuropsiquiátrica com posturas anormais, silêncio e estupor. A Colômbia tem uma prevalência de $11,4 \%$ de pacientes psiquiátricos. Objetivo: Discutir o caso clínico de uma mulher de 34 anos com uma história de transtorno depressivo maior que chega ao departamento de emergência com delírios niilistas e os sintomas catatônicos. Relato de caso: Foi relatado o caso de uma jovem adulta com transtorno depressivo maior e sintomas psicóticos que tinha sido hospitalizada nove meses antes, a quem deram alta com tratamento medicamentoso que ela não se lembrava. A paciente chegou ao pronto socorro do Hospital Universitário de Santander, apresentando três dias de um comportamento incomum, mutismo e negativismo. Exames laboratoriais e tomografia do crânio normal. O tratamento foi iniciado com uma benzodiacepina que a remeteu aos sintomas catatônicos. Posteriormente demonstrou anedonia, tristeza e delírios niilistas; considerou-se que seu estado correspondia a uma recorrência de episódio depressivo, começado nove meses antes, relacionado com a síndrome de Cotard. Gradualmente a paciente foi medicada com sertralina de $150,0 \mathrm{mg}$ a $5,0 \mathrm{mg}$ e olanzapina de $5.0 \mathrm{mg}$, obtendo a remissão dos sintomas afetivos e psicóticos. Discussão: $A$ desordem afetiva é a causa mais comum de catatonia. Há relatos semelhantes, embora poucos onde coexistam os três sintomas; este é o primeiro caso relatado no Hospital Universitário de Santander, com prévio consentimento informado. Conclusões: É incomum para uma pessoa deprimida ter delírios de negação e sintomas catatônicos simultaneamente, de modo que este caso, por ser único, gera novidade para a literatura. No caso, os sintomas catatônicos dificultam a exploração de outras esferas mentais e podem ser considerados secundários a uma condição médica, motivo pelo qual é essencial excluir causas orgânicas, tratá-los prontamente e assim esclarecer a psicopatologia subjacente. [Torrado-Arenas DM, Santos-Gutiérrez KE, Ruiz-Higuera SM, Zabala -Arias LM, Niño-García JA. Síndrome de Cotard associada ao transtorno depressivo maior com sintomas catatônicos. Relato de caso. MedUNAB 2016; 18 (1): 76-80].

Palavras-chave: Catatonia; Depressão; Negação (Psicologia);Antipsicóticos; Transtornos Psicóticos Afetivos.

son en orden de frecuencia: 1) trastornos afectivos, 2) esquizofrenia y 3 ) causas médicas (alteraciones metabólicas, infecciosas, endocrinas y neurológicas), estás últimas las de mayor mortalidad (1). La catatonia se puede medir a través de la escala de calificación de Bush Francis para catatonia (BFCRS), la cual evalúa la gravedad y la respuesta al tratamiento (7).

Por otra parte el síndrome de Cotard es una condición poco frecuente y su asociación a catatonia es aún más rara. Fue descrito por primera vez por el francés Jules Cotard en 1882 (7). Se caracteriza por presentar delirios de negación que van desde ideas nihilistas relacionadas con el cuerpo, siendo esta la más común, hasta la negación de su propia existencia y del entorno que la rodea $(7,8)$. Algunos estudios han encontrado una prevalencia, que es de menos del 1.0\% (2). Principalmente se ha visto asociado a: depresión, esquizofrenia y neurosífilis $(8,9)$. También se ha visto 
coexistente con delirios de Capgras y Licantropía $(1,7)$. En el estudio de Berrios GE et al, los principales síntomas que se observaron en una serie de 100 casos fueron: depresión $(89.0 \% \mathrm{~d} / \mathrm{c})$, delirios nihilistas $(69.0 \% \mathrm{~d} / \mathrm{c})$, ansiedad $(65.0 \% \mathrm{~d} / \mathrm{c})$, delirios de culpa $(63.0 \% \mathrm{~d} / \mathrm{c})$, delirios hipocondríacos $(58.0 \% \mathrm{~d} / \mathrm{c})$ y delirios de inmortalidad $(55.0 \% \mathrm{~d} / \mathrm{c})(2,10-12)$.

Según la teoría biológica la depresión se relaciona con una hipoactividad serotoninérgica y noradrenérgica. Respecto al componente psicótico se ha identificado un aumento de hormona liberadora de corticotropina y una desregulación de dopamina (13). Por otro lado, en la catatonía se ha encontrado alteración de la neurotransmisión gabaérgica (7). Finalmente se reportan alteraciones de estructuras anatómicas del lóbulo parietal derecho tanto en catatonia como en delirios nihilistas que explican su simultaneidad $(7,13)$.

Se han reportado casos similares pero pocos donde coexistan las tres entidades. Este es el primer caso reportado en el Hospital Universitario de Santander (HUS), con previo consentimiento informado. El objetivo es discutir el curso clínico de una mujer de 34 años con antecedente de trastorno depresivo mayor que llega de urgencias con delirios nihilistas y síntomas catatónicos.

\section{Reporte de caso}

Se trató de una mujer de 34 años, ama de casa, procedente de Bucaramanga, Colombia quien tenía antecedentes personales de maltrato intrafamiliar, abuso sexual en su adolescencia y antecedentes familiares de esquizofrenia en su madre y abuela. Además rasgos de personalidad evitativa y con miedo a eventos sociales.

Con cuadro que inició hace nueve meses caracterizado por tristeza durante la mayor parte del día, todos los días, llanto fácil, irritabilidad, anhedonia, hiporexia, ideas de minusvalía e insomnio de conciliación; sin ideas de muerte y suicidio. Tres semanas después de la presentación de los síntomas afectivos aparecieron alucinaciones auditivas complejas que la paciente describió como la voz de un hombre que le daba órdenes "escuché la voz de un hombre dentro de mi cabeza que me decía que la gente me iba a hacer daño a mí y a mis hijos, que tenía que defenderme, por eso insultaba y escupía a las personas", e ideas delirantes de tipo persecutorias que la llevaron a aislarse por temor a ser atacada, "mi familia me quería hacer daño y hacerle daño a mis hijos, ellos me los querían quitar, por eso me encerraba y me rasguñaba". Por este motivo se hospitalizó en dos oportunidades en una unidad de salud mental en Bogotá D.C y egresó con tratamiento farmacológico que la paciente no recordaba, con lo que se logró remisión completa de los síntomas psicóticos, pero la paciente afirmaba que continuaba experimentando labilidad emocional.
En el último mes, la paciente describió que se sentía triste la mayor parte del tiempo, sin interés por ninguna actividad que le gustaba hacer e insomnio de conciliación. Dos semanas antes de la hospitalización actual, la hermana de la paciente describió un comportamiento desorganizado "hace 15 días había menstruado y la encontré en la cama desnuda llena de sangre sosteniendo fuertemente a su hijo menor, diciendo que lo habían violado con un palo".

Así mismo tres días antes del ingreso a urgencias la hermana mencionó que la observó inmóvil por horas, en ocasiones con posturas extrañas, no respondió ninguna pregunta, se negó a recibir alimentos y cuando se le acercaban las personas las escupía. Motivo por el cual fue llevada por su hermana al servicio de urgencia del HUS, en la evaluación inicial la paciente se encontraba alerta, con notable descuido en su aseo y presentación personal (pelo enmarañado, uñas sucias, saliva en la cara y tórax), no colaboraba con la entrevista, permanecía mutista, y presentaba negativismo activo. Al examen físico sin alteración en signos vitales y sin otros hallazgos. Se decidió realizar tomografía simple de cráneo, punción lumbar, hemograma, electrolitos, serología, hormona estimulante de la tiroides, paraclínicos que se encontraron en rangos normales. Se decidió iniciar lorazepam $2.0 \mathrm{mg}$ cada ocho horas lográndose remisión de los síntomas catatónicos en el transcurso de los siguientes cinco días.

Es entrevistada nuevamente y mencionaba que días previos (mientras estuvo catatónica) escuchaba una voz que le impedía hablar "yo escuchaba una voz que me decía que no les podía responder nada o me castigaba". De la misma manera afirmaba que le habían quemado los ojos y que ya estaba muerta "no podía abrir los ojos porque me los habían quemado, estaba vacía y muerta por dentro, escupía para liberar mi alma y ser libre de este cuerpo sin vida".

Según el Manual diagnóstico y estadístico de los trastornos mentales (DSM-5) se consideró que la paciente presentaba un trastorno depresivo mayor con síntomas psicóticos, que incluían ideas nihilistas y se inició tratamiento con sertralina incrementando la dosis gradualmente hasta llegar a $150.0 \mathrm{mg}$ y olanzapina $5.0 \mathrm{mg}$ hasta llegar a $15.0 \mathrm{mg} /$ día, lográndose remisión de los síntomas. Se eligió esta combinación que según la literatura ha mostrado una eficacia mayor del $50.0 \%$, donde el antidepresivo potencia el antipsicótico. El control en el siguiente mes es con evolución satisfactoria. Continúa en seguimiento.

\section{Discusión}

Existen pocos reportes sobre la coexistencia de síndrome de Cotard, catatonia y depresión como lo ocurrido en el presente caso. Una vez resuelta la catatonia la exploración psicopatológica devela delirios nihilistas, por lo que se contempla la posibilidad de un síndrome de Cotard sobre un trastorno depresivo (14). 
En cuanto a los factores psicosociales, el antecedente de abuso sexual y maltrato físico predisponen al desarrollo de trastorno afectivo, como se ha evidenciado en el estudio de Dallos et al. que reporta que el $72.7 \%$ de las víctimas de abuso sexual tienen una complicación mental, donde la depresión ocupa el segundo lugar con un $25.0 \%$ de las complicaciones $(15,16)$. Al igual Ibarra et al. demuestra que de un grupo de mujeres con depresión, $76.0 \%$ de ellas habían sido víctimas de violencia física en la infancia (17). Otro aspecto a tener en cuenta son los rasgos de personalidad del grupo $\mathrm{C}$, especialmente los evitativos presentes en ella; con los cuales también se ha encontrado una estrecha relación con depresión(18).

Lo más importante en síndrome de Cotard y catatonia es descartar las causas orgánicas como infecciones y trastornos metabólicos $(7,8)$. En cuanto al manejo del caso, lo primordial es manejar primero la catatonia y luego los otros síntomas asociados, dado que la primera es una emergencia, por la posibilidad de inanición que le pone en situación de riesgo vital. Hay dos posibilidades de manejo que se han estudiado. La primera y más recomendada es el uso exclusivo de benzodiacepinas, especialmente el lorazepam, que es el medicamento de elección debido a su eficacia terapéutica que potencian la actividad gabaérgica en un $60.0 \%$ a $80.0 \%$ de los casos $(5,19,20)$.

La segunda opción es el uso de Terapia Electroconvulsiva con Anestesia y Relajación (TECAR) solo o en combinación con lorazepam, también eficaz en síntomas nihilistas (9). Lo anterior es debido a que no existen ensayos clínicos que nos muestren la verdadera eficacia del TECAR; dejándola solo para la catatonia refractaria, para la cual tiene eficacia cercana al $90.0 \%(19,21)$.

En el manejo de la catatonia, el uso de antipsicóticos de forma inicial para los síntomas psicóticos asociados está contraindicado, debido a que pueden causar un empeoramiento, al punto de desencadenar el síndrome neuroléptico maligno (6). Estos solo deben usarse, una vez resueltos los síntomas catatónicos y encontrando de base un trastorno psicótico.

El resto de componentes asociados al caso, de los cuales no hay claridad en el diagnóstico, se maneja después de la resolución de los síntomas catatónicos, debido a la imposibilidad de realizar la entrevista con el paciente en un primer momento. Luego de encontrar la causa de base, ya sea del estado del ánimo o de tipo psicótico, se da el antidepresivo o antipsicótico con mejor respuesta $(19,20,22)$.

El manejo del síndrome de Cotard se orienta a tratar la psicopatología de base, que en su mayoría es un trastorno depresivo (2). En adultos con diagnóstico de depresión con síntomas psicóticos se recomienda la combinación de antipsicótico y antidepresivo, generalmente donde el uno potencie al otro. Como la combinación sertralina y olanzapina que ha mostrado más efectividad que la sola monoterapia con antipsicótico; como el estudio de Meyers
B et al. que tuvo mayor remisión respecto a la monoterapia $(\mathrm{OR}=1.28,95 \% \mathrm{CI}=1.12-1.47, \mathrm{p}<0.001)(23-25)$.

Finalmente el pronóstico de la depresión psicótica es mejor que los trastornos esquizoafectivos o psicóticos primarios. Sin embargo, quienes han experimentado un episodio depresivo con síntomas psicóticos tienen un mayor riesgo de recaída y suicidio, en comparación con aquellos sin síntomas psicóticos (20). Por lo cual este caso sirve de referencia para otros estudios y debe hacerse control ambulatorio por riesgo de recaída. Este caso es único por la simultaneidad de tres entidades, tener buena respuesta al tratamiento y ser el primero reportado en nuestra institución.

\section{Conclusiones}

1. La catatonia es un síndrome que no solo se relaciona con esquizofrenia, de hecho los principales trastornos psiquiátricos de base asociados son los trastornos afectivos.

2. Por la dificultad para explorar si los pacientes con catatonia presentan psicopatología del espectro psicótico o afectivo es indispensable darles tratamiento oportuno con benzodiacepinas para orientar el diagnóstico y tratamiento específico.

3. Una gran proporción de la catatonia tiene causas orgánicas potencialmente mortales por lo cual es indispensable descartar estas condiciones médicas antes de atribuir su etiología a un trastorno psiquiátrico primario.

4. Según las teorías neurobiológicas los síntomas psicóticos tendrían sustrato en la alteración de dopamina y cortisol, mientras que los catatónicos en desregulación del GABA.

5. El tratamiento de elección para la catatonia son las benzodiacepinas, principalmente lorazepam. $\mathrm{Y}$ el uso de antipsicóticos debe ser cuidadoso por el riesgo de inducir síndrome neuroléptico maligno.

6. El pronóstico de los depresivos con síntomas psicóticos y catatónicos es peor por tener mayores tasas de recaída y riesgo suicida, cuando se compara con aquellos que solo presentan síntomas afectivos.

\section{Conflicto de intereses}

No hay conflicto de intereses para los autores.

\section{Referencias}

1. Worku B, Fekadu A. Symptom profile and short term outcome of catatonia: an exploratory clinical study. BMC Psychiatry 2015;15:164.

2. Debruyne H, Portzky M, Peremans K, Audenaert K. Cotard's Syndrome. MIND \& BRAIN, THE JOURNAL OF PSYCHIATRY $2011 ; 2(1): 67-72$.

3. Crespo ML, Pérez V. Catatonia: un síndrome neuropsiquiátrico. Revista Colombiana de Psiquiatría $2005 ; 24(2): 251-66$ 
4. Tandon R, Heckers S, Bustillo J, Barch DM, Gaebel W, Gur RE, et al. Catatonia in DSM-5. Schizophr Res 2013;150(1):26-30.

5. Sienaert P, Dhossche DM, Vancampfort D, De Hert M, Gazdag G. A clinical review of the treatment of catatonia. Front Psychiatry 2014;5:1-8.

6. Heckers S, Tandon R, Bustillo J. Catatonia in the DSM, Shall We Move or Not? Schizophrenia Bulletin 2010;36(2):205-07.

7. Basu A, Singh P, Gupta R, Soni S. Cotard Syndrome with catatonia: Unique Combination. Indian Journal of Psychological Medicine 2013;35(3):314-16.

8. Machado L, Peregrino A, Azoubel S, Cerqueira H, Lima Filho L. Cotard's syndrome and major depression with psychotic symptoms. Rev Bras Psiquiatr 2013;35:212.

9. Weiss C, Santander J, Torres R. Catatonia, Neuroleptic Malignant Syndrome, and Cotard Syndrome in a 22-YearOld Woman: A Case Report. Case Reports in Psychiatry 2013;1:1-3.

10. Grover S, Aneja J, Mahajan S, Varma S. Cotard's syndrome: Two case reports and a brief review of literature. J Neurosci Rural Pract 2014;5(1):S59-62.

11. Mustafa Özköse M, Havle N, Saygin Sari I, Özgen G. Cotard Sendromu: Ender Rastlanan Bir Vak'a. Academic Journal 2010;48(2):129-31.

12. Basu A, Singh P, Gupta R, Soni S. Cotard syndrome with catatonia: unique combination. Indian J Psychol Med 2013;35(3):314-6.

13. Nelson E. Psychotic Depression beyond the Antidepressant/Antipsychotic Combination. Curr Psychiatry Rep 2012;14:619-23.

14. American Psychiatric Association. Manual diagnóstico y estadístico de los trastornos mentales. DSM-IV. 4th ed. Washington: APA; 2001.

15. Lara MA, Navarrete L, Nieto L, Le HN. Childhood abuse increases the risk of depressive and anxiety symptoms and history of suicidal behavior in Mexican pregnant women. Rev Bras Psiquiatr 2015;29:1-9.

16. Dallos Arenales MI, Pinzón Amado A, Barrera González C, Mujica Rojas J, Meneses Silva Y. Impacto de la violencia sexual en la salud mental de las víctimas en Bucaramanga, Colombia. Rev. Colombiana Psiquiatría 2008;37(1):56-65

17. Ibarra Alcantar MC, Ortiz Guzmán JA, Alvarado Cruz FJ, Graciano Morales H, Jiménez Genchi A. Correlatos del maltrato físico en la infancia en mujeres adultas con trastorno distímico o depresión mayor. Salud Ment 2010;33(4):317-24.

18. Van Rijsbergen G, Kok G, Elgersma H, Hollon S, Bockting $\mathrm{C}$. Personality and cognitive vulnerability in remitted recurrently depressed patients. Journal of Affective Disorders 2015;173:97-104.

19. Raveendranathan D, Narayanaswamy J, Reddi S. Response rate of catatonia to electroconvulsive therapy and its clinical correlates. Eur Arch Psychiatry Clin Neurosci 2012;262:425-30.

20. Rosebush P, Mazurek M. Catatonia and Its Treatment. Schizophrenia Bulletin 2010;36(2):239-42.

21. Dhossche DM, Sienaert $P$, van der Heijden FM. Mechanisms of catatonia. Tijdschr Psychiatr. 2015;57(2):99-103.
22. Francis A. Catatonia: Diagnosis, Classification, and Treatment. Curr Psychiatry Rep 2010;12:180-85.

23. Meyers B, Flint A, Rothschild A, Mulsant B. A double-blind randomized controlled trial of olanzapine plus sertraline versus olanzapine plus placebo for psychotic depression. Arch Gen Psychiatry 2009;66(8):838-47.

24. Bhattacharya S, GagliardiJP. Walking corpse syndrome presenting with catatonia: Case report and literature review. Biological Psychiatry 2015;77(9):263S-64S.

25. Mendez MF, Ramírez-Bermúdez J. Cotard syndrome in semantic dementia. Psychosomatics 2011;52(6):571. 